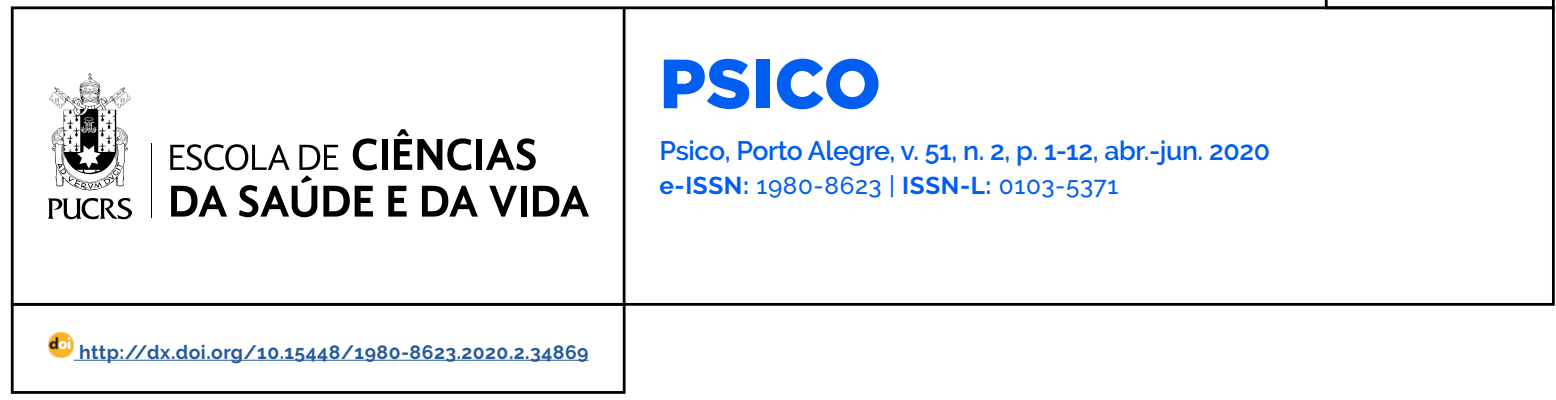

SEÇÃO: ARTIGO

\title{
Transição para educação infantil: estudo comparativo do processo de vinculação primária e secundária
}

\author{
Transition to childhood education: a comparative study of the primary and secondary \\ bonding process
}

Transición a la educación infantil: un estudio comparativo del proceso de vinculación primaria y secundaria

Isabela Rocha Izidoro ${ }^{1}$ orcid.org/0000-0003-3612-2387 isabel.ai.zidoro@hotmail.com

\section{Veronica Aparecida \\ Pereira ${ }^{1}$}

orcid.org/0000-0002-9051-7550 veronica.ufgd.tci@gmail.com

\section{Olga Maria Piazentin Rolim Rodrigues ${ }^{2}$} orcid.org/0000-0002-5332-256X olgarolim@uol.com.br

Recebido em: 16 jul. 2019. Aprovado em: 25 fev. 2020. Publicado em: 4 set. 2020.

\section{(c) (i)}

Artigo está licenciado sob forma de uma licença Creative Commons Atribuição 4.0 Internacional.
Resumo: O presente artigo buscou: 1) descrever e comparar: a) a qualidade do vinculo entre o bebê-mãe e bebê-educadora, aos nove e aos doze meses de idade; b) a percepção do temperamento do bebê pelas mães e pelas educadoras e, 2) correlacionar os indicadores de vinculação com os escores obtidos nas escalas de temperamento. Participaram nove bebês e suas respectivas mães e duas educadoras. Mães e educadoras responderam a escala de temperamento e foram filmadas individualmente com os respectivos bebês, aos nove e aos doze meses. Os resultados indicaram a presença de vinculação primária com a mãe e secundária com a educadora, ambas satisfatórias. Houve correlações negativas entre temperamento dificil do bebê e comportamentos interativos maternos. Embora o vínculo mãe-bebê seja mais forte, a qualidade estabelecida com as educadoras sugere que a transição para a educação infantil pode ser positiva.

Palavras-chave: vinculação primária, vinculação secundária, apego, relação mãe-bebê, educação infantil.

Abstract: This paper aims: 1) to describe and to compare: a) the quality of the bond between a baby-mother and a baby-educator, at nine and twelve months old; b) the perception of the babies' temperament by mothers and by educators and, 2) c) to correlate the bonding indicators with scores in temperament scales. Participated nine babies, their mothers and two teachers. Mothers and educators answered the temperament scale and were filmed individually with their respective babies, at nine and twelve months. The results indicated the presence of a primary bond with the mother and a secondary bond with the educator, both satisfactory. There were negative correlations between the baby's difficult temperament and maternal interactive behaviors. Although the mother-baby bond is stronger, the quality established with the educators suggests that the transition to early childhood education can be positive.

Keywords: primary attachment, secondary attachment, attachment, mother-baby relationship, child education.

Resumen: El presente artículo buscó: 1) describir y comparar: la calidad del vinculo entre el bebé y su madre y su educadora, a los nueve y doce meses de vida; b) la percepción del temperamento del bebé según las madres y según las educadoras y, c) correlacionar las categorias de conductas de vinculación a los resultados obtenidos en las escalas de temperamento. Participaron nueve bebés y sus respectivas madres y dos educadores. Madres y educadores respondieron a la escala de temperamento y fueron filmadas individualmente con sus respectivos bebés, a los nueve y doce meses. Los resultados indicaron la presencia de vinculación primaria con la madre y secundaria con la educadora, ambas satisfactorias. Hubo correlaciones negativas entre el temperamento difícil del bebé y los comportamientos interactivos maternos. Aunque el vínculo 
madre-bebé es más fuerte, la calidad establecida con los educadores sugiere que la transición a la educación de la primera infancia puede ser positiva.

Palabras clave: apego primario, apego secundario, apego, relación madre-bebé, educación infantil.

No presente estudo, ${ }^{3}$ tanto o processo de vinculação primária como secundária são avaliados em uma perspectiva bidirecional, na qual entende-se que o comportamento do adulto e da criança se influenciam mutuamente. O bebê, desde o nascimento, traz consigo caracteristicas individuais importantes, que passam a ser percebidas pelo adulto como sinais de temperamento, sendo alguns desses sinais mais favoráveis a interação e outros menos.

Desse modo, os tópicos seguintes discorrem acerca de estudos sobre os processos de vinculação primária e secundária e possiveis relações com o temperamento infantilna percepção do adulto. Nesse contexto, os processos de vinculação, avaliados a partir dos comportamentos interativos observados aos nove e aos doze meses, e a percepção do adulto sobre o temperamento dos bebês são correlacionados entre si.

\section{O afeto como fator de proteção: processos de vinculação primária e secundária}

Dentre os fatores de proteção para o desenvolvimento infantil comumente estudados encontram-se o desenvolvimento de uma gestação saudável, o nascimento a termo, boas condições de parto, a responsividade materna, a saúde emocional materna durante a gestação e no pós-parto, a rede de apoio para mãe e o bebê, os ambientes com estimulação adequada e suficiente, assim como a boa vinculação e os processos de interação satisfatórios entre cuidador-bebê que, na maioria das pesquisas, caracteriza-se pela mãe (Chiodelli, 2016; Pereira, Rodrigues, Carvalho, \& Chiodelli, 2015). Nessa direção, Saur, Bruck, Antoniuk e Riechi (2018) indicaram que o estabelecimento de vinculações primárias seguras são preditoras para o bom desenvolvimento tanto emocional como cognitivo.
Entende-se aqui como vinculação primária, aquela estabelecida primariamente com aquele que era seu cuidador principal, antes de exercer cuidados compartilhados com outros adultos. Segundo Ainsworth, Blehar, Waters e Wall (1978) uma boa vinculação primária pode, posteriormente, favorecer a gestão de futuras relações e orientar ações em situações sociais exteriores à família. Mais comumente, a vinculação primária tende a ocorrer com a figura materna, visto que a mãe, na maioria das vezes, é a primeira a ofertar, de forma constante, cuidados ao bebê. Nardi, Rodrigues, Melchiori, Salgado e Tavano (2015) indicaram que a vinculação primária se estabelece desde o primeiro contato da mãe com o bebê, a partir das interações estabelecidas entre a diade, permeada pelas muitas atividades típicas da idade como amamentar, trocar, dar banho, acalentar, entre outras.

Montirosso et al. (2017) compararam os processos de vinculação e autorregulação com uma amostra de 50 diades mãe-bebê, distribuidas em dois grupos ( 25 de bebês nascidos a temos e 25 de bebês prematuros). O estudo, pautado no paradigma Face to Face Still-Face (FFSF) (Tronick, Als, Adamson, Wise, \& Brazelton, 1978), indicou que os bebês prematuros apresentaram mais comportamentos de protesto (afastarse, torcer-se arquear o corpo) durante o FFSF. Além disso, durante e depois do episódio não interativo, apresentaram mais comportamentos de autorregulação.

Seixas et al. (2017) descreveram as contribuições do comportamento materno para autorregulação de bebês, em pesquisa desenvolvida com 98 diades formadas por bebês nascidos a termo. No estudo, os autores indicaram que, entre as diversas variáveis maternas que se relacionam com a autorregulação, têm destaque o modo como a mãe se relaciona com o bebê, o número de filhos, o índice de Apgar e o sexo do bebê.

Para além das variáveis maternas, as relações de vinculação precisam ser compreendidas

3 O artigo apresenta dados oriundos da dissertação de autora Isabela Rocha Izidoro, defendida junto ao Programa de Psicologia UFGD, em 2019, com bolsa Fundect-Capes, intitulada Análise comparativa do desenvolvimento da vinculação mãe-bebê e educadora-bebê: contribuições para o desenvolvimento infantil. 
também em torno de outras figuras. Bowlby (1989) indicou que a vinculação pode ser estabelecida com a mãe ou outra figura que exerça o papel parental, estabelecendo proximidade, aconchego e segurança. O papel exercido pelas educadoras é diferente do papel parental tendo, muitas vezes, a proximidade prejudicada pela presença de muitas crianças sob responsabilidade de um mesmo educador. Nesse contexto, alguns estudos investigaram as relações estabelecidas entre o bebê e outros cuidadores (vinculação secundária) comparando ao processo de vinculação estabelecido com o cuidador principal, na maioria das vezes, a mãe (vinculação primária) (Farran \& Ramey, 1977; Bowlby, 1989; Caruso, 1990; Howes \& Spieker, 2016; Baumgartner, Bellucci \& Laghi, 2017; Cárcamo, García-Riquelme, Lagos, \& Gómez, 2019).

Farran e Ramey (1977) discutiram, a partir de uma pesquisa realizada em centros de educação infantil, o estabelecimento da vinculação dos bebês com as educadoras em comparação a vinculação estabelecida com a mãe. Segundo eles, embora possa ocorrer a formação de outros processos de vinculação, o processo inicial com a mãe não é afetado. Entretanto, esses processos secundários não serão tão fortes como o primeiro, já previamente estabelecido.

A perspectiva de Caruso (1990), a partir de um referencial da educação infantil, sugere que os bebês, ao terem seus cuidados compartilhados entre dois ou mais adultos, a mãe e as educadoras, têm seus processos de vinculação primária fragilizados. Esse autor sustenta que há formação de processo de vinculação entre o bebê e a educadora, porém, ao acontecer, pode influenciar negativamente a manutenção do processo de vinculação primário já estabelecido com a mãe.

Tanto Farran e Ramey (1977) como Caruso (1990) defendem que os processos de vinculação secundária, prejudicam e/ou enfraquecem a vinculação primariamente estabelecida, ou que não serão tão fortes quanto a primeira. Uma das hipóteses pode estar relacionada ao fato do tipo de relação afetiva estabelecida entre as crianças e suas mães ser mais forte do que as relações de afeto com outros adultos.
Cárcamo, García-Riquelme, Lagos e Gómez (2019) realizaram um estudo com 24 diades sobre a vinculação de bebês frequentadores de creches e suas mães. Utilizaram protocolos para registro de comportamentos maternos e dos bebês acerca da qualidade da vinculação, tendo como base o procedimento experimental da Situação Estranha de Ainsworth (Ainsworth, Biehar, Waters, \& Wall, 1978). Utilizaram também a Escala de Apego durante Estresse (ADS) (Massie \& Campbell, 1983). Os bebês tinham entre seis e dez meses de idade. Os resultados apontaram maiores percentuais de bebês com apego seguro (68\%), o que, segundo os autores, confirma os achados anteriores com populações chilenas. Correlações entre dados sociodemográficos indicaram relações positivas entre apego seguro e idade de entrada na educação infantil, indicando que os bebês que ingressaram mais tarde apontaram maiores indices para apego seguro. Correlações negativas foram apontadas entre o tempo de permanência em sala de aula e apego seguro, sendo melhores os resultados para os bebês que permaneciam menos tempo longe de seus pais.

Por outro lado, o estudo de Howes e Spieker (2016) indicou que o número de horas que as crianças passam em creches apresenta forte associação com o tipo de apego que se estabelece com o educador. Nessa direção, Baumgartner, Bellucci e Laghi (2017) destacaram que não só é possivel estabelecer um padrão de apego seguro com os cuidadores alternativos como esse não interfere no tipo de apego estabelecido com os pais.

Investigações com populações brasileiras podem contribuir para um conhecimento mais detalhado sobre os processos de vinculação primária e secundária, a fim de favorecer o processo de transição e permanência na educação infantil como uma forte contribuição para o desenvolvimento infantil e das relações parentais. Essa investigação é de fundamental importância, considerando que a função de cuidados dos filhos não pode ser exclusiva da mãe, devendo ser compartilhada com demais familiares (rede de apoio) e outros adultos (educadores e outros profissionais). 
Cientes da importância do vínculo para o desenvolvimento infantil, discute-se, a partir de mudanças sociais relacionadas aos papeis familiares, como favorecer a oferta de cuidados para a criança de modo a viabilizar padrões de vinculação como fatores de proteção e autorregulação (Montirosso, Borgatti, Trojan, Zanini, \& Tronick, 2010; Seixas, Barbosa, \& Fuertes, 2017).

No estudo de Tronick (2007) observou-se como o estado emocional das mães e as representações que elas têm sobre o filho influenciam a forma como reagem às suas solicitações. Nestes contextos, faz-se necessário reconhecer como os adultos, e não apenas as mães, percebem os bebês, em termos de características individuais, como o temperamento, e como essa percepção pode interferir nos processos de vinculação.

\section{O temperamento de crianças pequenas - a percepção dos adultos}

O termo temperamento tem definido os estilos comportamentais diferentes observados em humanos desde muito cedo (Sanson, Hemphill, \& Smart, 2004). Thomas e Chess (1977) propuseram uma classificação de temperamento infantil em: fácil, difícil e de aquecimento lento. Crianças de temperamento fácil tendiam a adaptar-se rapidamente a novas demandas do ambiente. Aquelas com temperamento difícil apresentavam dificuldades de adaptação a novas situações e humor negativo e, as crianças cujo temperamento era classificado como de aquecimento lento apresentavam dificuldades iniciais de adaptação, mas acabavam respondendo positivamente às novas demandas.

Entre as diferentes definições de temperamento é possivel considerá-lo como uma variável hereditária, na qual podem ser identificadas respostas emocionais básicas como medo, raiva e apego (Costa, Thomaz, Araújo, Medeiros, \& Galdino, 2019), mas, também comportamentos positivamente adaptativos como simpatia e acolhimento que, ao longo do desenvolvimento, passam por influências socioculturais, mas que determinam a adaptabilidade ou não a novas situações. Outros estudos pautaram-se no autorrelato do adulto acerca de respostas comportamentais da criança frente ao ambiente, ou observação sistemática de uma situação estruturada (Fuertes, Lopes dos Santos, Beeghly, \& Tronick, 2009; Malhado \& Alvarenga, 2012; Chiodelli, 2016).

$\mathrm{Na}$ hipótese de autorrrelato, é mais comum que os adultos associem o choro excessivo e a irritabilidade ao temperamento dificil; e padrões de respostas de maior exploração, sociabilidade e de receptividade estariam entre temperamento fácil ou moderado. Contudo, é importante considerara via bidirecional, ou seja, como o adulto responde aos padrões apresentados pelos bebês, em termos de receptividade, acolhimento e respostas contingentes às suas necessidades (Chiodelli, 2016).

Entre as associações observadas nas relações diádicas, observou-se que os bebês cujas mães relataram temperamento como mais positivo, tendem a desenvolver um vínculo seguro com as suas mães durante o primeiro ano de vida (Fuertes, Lopes dos Santos, Beeghly, \& Tronick, 2009) podendo essa variável ser preditiva para vínculo seguro, o que implica em condições mais favoráveis de desenvolvimento na primeira infância.

Contudo, são ainda poucos os estudos que avaliam a vinculação com terceiros sobre o processo de vinculação entre os bebês e suas mães. Por isso, no presente estudo buscou-se: a) descrever e comparar a qualidade do vínculo entre o bebê e sua mãe e entre o bebê e sua educadora, aos nove e aos doze meses de vida; b) descrever e comparar a percepção do temperamento do bebê pelas mães e pelas educadoras aos nove meses de vida do bebê e, c) correlacionar as categorias de comportamentos de vinculação observados aos nove e aos doze meses aos escores obtidos nas escalas de temperamento respondidas pelas mães e pelas educadoras.

\section{Método}

Trata-se de um estudo descritivo, observacional e longitudinal, aprovado pelo Comitê de Ética da Universidade Federal da Grande Dourados, Parecer $n^{\circ} 2.421 .238$. 


\section{Participantes}

A amostra da pesquisa foi constituída por nove pares bebê-mãe e nove pares bebê-educadora (embora fossem apenas duas educadoras, elas foram pareadas repetidas vezes garantindo que cada bebê fosse filmado com sua respectiva educadora). Para as diades mãe-bebê, cada bebê foi filmado com sua respectiva mãe aos nove e aos doze meses, enquanto oito bebês foram filmados com a mesma educadora (a qual prestava os cuidados principais) e um bebê com outra educadora (por ser de outro centro de educação infantil). As filmagens aconteceram em sala individual, por diade. As mães que participaram do estudo ( $\mathrm{N}=9$ ) tinham idade entre 22 e 30 anos (Mediana - Md = 27 Desvio Padrão - DP 2,774) e escolaridade entre 14 e 23 anos (Md 19; DP 2,828). Os bebês tinham idade gestacional entre $33 \mathrm{e}$ 41 semanas (Md 38; DP 2,848), peso ao nascer entre 1905 e 3855 gramas (Md 3.005; DP 0,605) e comprimento entre 43 e 53 centímetros (Md 47: DP 3,073). As duas educadoras tinham idade de 44 e 33 anos, com formação em Pedagogia, especialização e experiência profissional há pelo menos quatro anos.

\section{Local}

As filmagens ocorreram, na maioria, realizadas no Centro de Educação Infantil (CEI) frequentado pelos bebês, em horário previamente estabelecido. Apenas uma das díades solicitou que a filmagem ocorresse em ambiente domiciliar.

\section{Instrumentos}

Inicialmente, para coleta dos dados sociodemográficos, utilizou-se uma entrevista semiestruturada com a mãe e com as educadoras, a fim de levantar dados relevantes do contexto familiar e educacional.

Para a avaliação do temperamento utilizou-se a Escala de Temperamento dos Bebês (Fuertes, 2004). Trata-se de uma escala de autoaplicação, com nove questões do tipo Likert, com pontuações de um a sete, sendo as pontuações maiores indicativos de temperamento mais dificil.

A avaliação do processo de vinculação foi avaliada de duas formas: 1) Aos nove meses utilizou-se o protocolo de Comportamentos Maternos e do Bebê observados durante um procedimento experimental pautado no paradigma do Face-to-face Still Face (FFSF) (Tronick et al., 1978) estruturado a partir das categorias apresentadas por Fuertes (2004). Nos protocolos, as categorias de Orientação Social Positiva (OSP) (verbalizações, sorrisos, contato físico e visual) e Orientação Social Negativa (OSN) (como protestos e verbalizações negativas) foram registradas para o adulto e para o bebê. $O$ protocolo do bebê continha também descritores de Autorregulação (AUR). Durante a filmagem foram utilizados: cadeiras para bebê, filmadoras digitais e tripés; 2) Aos doze meses, utilizou-se a situação de interação livre, visto que nessa idade dificilmente se consegue manter os bebês em longo tempo em cadeirinhas. Utilizou-se para tanto o Sistema de Codificação da Interação Mãe-Criança Revisado (CITMI-R) (Alvarenga \& Cerezo, 2013). O CITMI-R é estruturado a partir de quatro categorias gerais (duas referentes ao comportamento da mãe ou responsável e duas ao comportamento da criança) classificadas em positiva, negativa e neutra e, não interativa). Tanto o procedimento dos nove meses como dos doze meses teve por objetivo a avaliação dos comportamentos presentes durante o processo de vinculação do bebê com o adulto.

\section{Procedimento}

A pesquisadora entrou em contato com as mães e com as educadoras e convidou-as explicando os objetivos da pesquisa e observando os procedimentos éticos. A seguir, solicitou o preenchimento da Escala de Temperamento, pelas educadoras e pelas mães, individualmente.

Para a filmagem da interação, aos nove meses, a pesquisadora assegurou que os bebês estivessem em condição confortável (alimentados, limpos, sem sinais de sono ou cansaço). Os episódios foram filmados em dois diferentes ângulos, sendo três episódios, de até três minutos cada, sinalizados pela pesquisadora quanto ao tempo. Durante a filmagem, o bebê 
permaneceu sentado em uma cadeirinha de alimentação, face a face com o adulto, mantendo contato visual e comunicação durante o primeiro e o terceiro episódio. No segundo episódio, o adulto manteve apenas contato visual. Sinais de choro por 15 segundos consecutivos, interrompiam o episódio (no segundo episódio) ou a filmagem (no caso de ocorrer no primeiro ou terceiro episódios).

Para a análise da interação livre, aos doze meses, foram disponibilizados alguns brinquedos, em colchonetes forrados, para que a mãe e a educadora, separadamente, pudessem sentar-se ao lado do bebê e interagir livremente durante cinco minutos. Nesse período a pesquisadora saiu da sala e deixou uma câmera ligada, controlando o tempo de interação. O posicionamento da câmera foi ajustado para capturar simultaneamente a imagem de ambos.

\section{Análise de dados}

Os dados obtidos foram analisados por meio do software estatístico SPSS (IBM, versão 24). Foram utilizadas estatísticas descritivas e testes não paramétricos. Em função do tamanho e características amostrais, utilizou-se o teste de Wilcoxon para as comparações e o teste de Spearman para as correlações.

As filmagens do FFSF passaram por edição para exibição simultânea, com a utilização do software Adobe Premiere Pro CS6. Os vídeos foram analisados quanto à ocorrência de comportamentos de OSP, OSN e AUR a cada cinco segundos, registrados no protocolo, por dois juizes independentes, com análise de concordância 0,836. A análise da interação livre foi realizada a partir da utilização do protocolo CITMI-R (Alvarenga \& Cerezo, 2013), também por dois juizes, com indice de concordância de 0,901.

\section{Resultados}

Para responder aos objetivos propostos os resultados encontram-se organizados em três seções: 1) avaliação do processo de vinculação aos nove meses - comparação da vinculação primária e secundária a partir do FFSF; 2) avaliação do processo de vinculação aos doze meses - comparação da vinculação primária e secundária a partir dos escores de interação do CITMI-R; e 3) correlações entre o processo de vinculação e a percepção de temperamento do bebê pelos adultos.

Avaliação do processo de vinculação aos nove meses - comparação da vinculação primária e secundária a partir do FFSF. Primeiramente, apresentam-se os dados descritivos do processo de vinculação observados aos nove meses e a comparação das categorias observadas para os comportamentos dos bebês e suas respectivas mães e educadoras, durante o primeiro e terceiro episódio do FFSF (antes e depois do episódio não interativo). Conforme observa-se na Tabela 1, os bebês apresentaram medianas semelhantes de Orientação Social Positiva (OSP) em interação com a mãe e com a educadora durante os três episódios. Já com relação às medianas de Orientação Social Negativa (OSN), os bebês, após o segundo episódio (não interativo), apresentaram medianas significativamente maiores com sua mãe do que com a educadora $(p=0,043)$, indicando que, após o episódio de estresse, protestaram mais com suas mães do que com as educadoras. Também, no terceiro episódio, os bebês apresentaram significativamente maiores medianas de comportamentos de autorregulação (AUR) com a educadora, do que com suas mães ( $p=0,043)$, sugerindo que os bebês apresentaram mais comportamentos não interativos com a educadora. 
Tabela 1 - Comparação entre as categorias do FFSF do bebê por episódio em interação com a mãe e com a educadora.

\begin{tabular}{|c|c|c|c|c|c|c|}
\hline & & \multicolumn{2}{|c|}{ FFSF bebê com a mãe } & \multicolumn{2}{|c|}{ FFSF bebê com a educadora } & \multirow[b]{2}{*}{$P$} \\
\hline & $N=8$ & Mediana (DP) & Min-Máx & $\begin{array}{l}\text { Mediana } \\
\text { (DP) }\end{array}$ & Min-Máx & \\
\hline \multirow{3}{*}{ OSP } & $1^{\circ}$ episódio & $1,545(0,843)$ & $0,72-3,31$ & $1,120(0,178)$ & $0,91-1,44$ & 0,499 \\
\hline & $2^{\circ}$ episódio & $0,810(0,356)$ & $0,00-1,16$ & $0,680(0,259)$ & $0,57-1,33$ & 0,686 \\
\hline & $3^{\circ}$ episódio & $1,410(0,699)$ & $0,00-2,00$ & $1,380(0,204)$ & $1,00-1,55$ & 0,686 \\
\hline \multirow{3}{*}{ OSN } & $1^{\circ}$ episódio & $0,160(0,429)$ & $0,00-1,16$ & $0,000(0,401)$ & $0,00-1,00$ & 0,463 \\
\hline & $2^{\circ}$ episódio & $0,880(0,848)$ & $0,41-3,00$ & $0,520(1,127)$ & $0,00-3,13$ & 0,225 \\
\hline & $3^{\circ}$ episódio & $1,120(0,931)$ & $0,13-3,00$ & $0,410(1,269)$ & $0,00-3,67$ & 0,043 \\
\hline \multirow{3}{*}{$A \cup R$} & $1^{\circ}$ episódio & $0,985(0,262)$ & $0,38-1,16$ & $0,760(0,211)$ & $0,32-1,02$ & 0,310 \\
\hline & $2^{\circ}$ episódio & $1,080(0,351)$ & $0,88-1,90$ & $1,380(0,205)$ & $1,25-1,85$ & 0,080 \\
\hline & $3^{\circ}$ episódio & $0,250(0,278)$ & $0,00-0,66$ & $0,880(0,498)$ & $0,47-2,00$ & 0,043 \\
\hline
\end{tabular}

Fonte: elaborada pela autora. Teste de Wilcoxon para duas amostras relacionadas. $p \leq 0,05$

Para análise da qualidade do vinculo compararamse os escores do primeiro e terceiro episódio do FFSF dos comportamentos das mães e das educadoras. A partir do teste de Wilcoxon, apresentado na Tabela 2, observa-se que não houve diferença significativa entre os as medianas registradas para mães e educadoras, sugerindo que elas se comportaram de modo semelhante durante o FFSF, tanto em relação aos comportamentos considerados positivos quanto aos negativos.

Tabela 2 - Comparação entre as categorias do processo de vinculação observadas para mãe-bebê $x$ educadora-bebê no primeiro e terceiro episódio do FFSF

\begin{tabular}{|c|c|c|c|c|c|c|}
\hline & & \multicolumn{2}{|c|}{$F F S F$ mãe $(\mathbf{n}=\mathbf{8} / \mathbf{8})$} & \multicolumn{2}{|c|}{$F F S F$ educadora $(n=2 / 8)$} & \multirow[b]{2}{*}{$P$} \\
\hline & & Mediana (DP) & Min-Máx & $\begin{array}{l}\text { Mediana } \\
\text { (DP) }\end{array}$ & Min-Máx & \\
\hline \multirow{2}{*}{ OSP } & $1^{\circ}$ episódio & $3,110(0,202)$ & $2,75-3,36$ & $2,895(0,477)$ & $1,97-3,45$ & 0,172 \\
\hline & $3^{\circ}$ episódio & $3,050(0,349)$ & $2,50-3,57$ & $3,020(0,432)$ & $2,27-3,66$ & 0,500 \\
\hline \multirow{2}{*}{ OSN } & $1^{\circ}$ episódio & $0,065(0,073)$ & $0,00-0,19$ & $0,000(0,056)$ & $0,00-0,16$ & 0,246 \\
\hline & $3^{\circ}$ episódio & $0,000(0,371)$ & $0,00-1,00$ & $0,000(0,093)$ & $0,00-0,25$ & 0,655 \\
\hline
\end{tabular}

Fonte: elaborada pela autora. Teste de Wilcoxon para duas amostras relacionadas. $p \leq 0,05$

\section{Avaliação do processo de vinculação aos doze} meses - comparação da vinculação primária e secundária a partir dos escores de interação do CITMI-R. A comparação das categorias de vinculação aos doze meses, contida na Tabela
3. por meio do protocolo CITMI-R, mostra que os bebês não apresentaram diferenças significativa em interação com a mãe e com a educadora. E, assim, aos doze meses, as duas também emitiram comportamentos semelhantes. 
Tabela 3 - Comparação entre as categorias de interação observadas em situação livre, a partir do CITMI-R, aos 12 meses, para as diades mãe-bebê x educação bebê.

\begin{tabular}{|c|c|c|c|c|c|c|}
\hline & & \multicolumn{2}{|c|}{ Mãe (n=7/7) } & \multicolumn{2}{|c|}{ Educadora $(n=7 / 2)$} & \multirow[b]{2}{*}{$P$} \\
\hline & & Mediana (DP) & Min-Máx & $\begin{array}{l}\text { Mediana } \\
\text { (DP) }\end{array}$ & Min-Máx & \\
\hline \multirow[t]{3}{*}{ Bebes } & $A^{+}$ & $3,000(3,952)$ & $0,00-11,00$ & $3,00(3,625)$ & $0-10$ & 0,348 \\
\hline & Ao & $11,000(14,931)$ & $6,00-44,00$ & $27,00(19,258)$ & $10-70$ & 0,498 \\
\hline & A- & $0,000(0,378)$ & $0,00-1,00$ & $0,00(0,755)$ & $0-2$ & 0,655 \\
\hline \multicolumn{2}{|c|}{ Total Interativos } & $16,000(17,688)$ & $9,00-55,00$ & $31,00(18,515)$ & $13-73$ & 0,463 \\
\hline & NRo & $59,000(12,134)$ & $29,00-66,00$ & $59,00(19,439)$ & $42-404$ & 0,237 \\
\hline \multirow[t]{3}{*}{ Adulto } & $\mathrm{S}^{+}$ & $26,000(12,065)$ & $13,00-49,00$ & $10,00(17,420)$ & $1-53$ & 0,176 \\
\hline & So & $58,000(8,750)$ & $41,00-67,00$ & $56,00(4,231)$ & $52-63$ & 0,833 \\
\hline & S- & $3,000(2,116)$ & $0,00-5,00$ & $0,00(0,377)$ & $0-1$ & 0,068 \\
\hline \multicolumn{2}{|c|}{ Total Interativos } & $86,000(18,243)$ & $60,00-111,00$ & $62,00(20,599)$ & $59-116$ & 0,237 \\
\hline & NR & $3,000(6,550)$ & $0,00-19,00$ & $5,00(6,890)$ & $3-18$ & 0,340 \\
\hline
\end{tabular}

Fonte: elaborada pela autora. Teste de Wilcoxon para duas amostras relacionadas. $p \leq 0,05$

\section{Correlações entre o processo de vinculação e a} percepção de temperamento do bebê pelo adulto.

Com relação às características temperamentais identificadas pela mãe e pela educadora, as educadoras indicaram com maior frequência que os bebês têm temperamento fácil (55,6\%), enquanto apenas $12,5 \%$ das mães identificaram seus bebês nessa categoria. Já na categoria temperamento moderado, observou-se o percentual de 75,0\% das mães e 33,3\% para as educadoras. As mães e as educadoras apresentaram valores semelhantes quanto ao número de bebês com temperamento dificil $(12,5 \%$ e $11,1 \%)$.

Os escores brutos obtidos por meio da escala de temperamento, respondidos pelas mães e pelas educadoras foram comparados por meio do teste de Wilcoxon, com nivel de confiança de 95\%, porém, não houve diferença significativa.

Para a análise correlacional, conforme observase na Tabela 4, as categorias de vinculação do FFSF e do CITMI-R foram correlacionadas com os escores brutos da escala de temperamento indicados pelas mães e pelas educadoras. Para o FFSF utilizou-se o primeiro episódio, por ainda não sofrer influência do episódio não interativo. Os escores brutos de temperamento indicados pelas educadoras apresentaram correlação forte e positiva com a OSN do bebê em interação com a educadora $(p=0,018)$. Não houve diferença significativa entre os indicadores de temperamento pelas mães e as categorias de vinculação, embora o resultado da correlação de $p=0,054$ sugira uma tendência à correlação entre maiores escores de temperamento correlatos a mais comportamentos de orientação negativa do bebê. Os escores de temperamento indicados pelas educadoras apresentaram mais três correlações muito fortes e negativas, com a categoria $A+(p=0,015), S+$ $(p=0,027)$ e com o total de comportamentos interativos positivos da educadora $(p=0,031)$. 
Tabela 4 - Correlações significativas entre as categorias de vinculação do FFSF e do CITMI-R com os escores de temperamento indicados pela educadora e pela mãe

\begin{tabular}{|c|c|c|c|c|}
\hline & Correlações & & $\begin{array}{c}\text { Escores de } \\
\text { Temperamento } \\
\text { indicados pela } \\
\text { educadora }\end{array}$ & $\begin{array}{c}\text { Escores de } \\
\text { Temperamento } \\
\text { indicados pela mãe }\end{array}$ \\
\hline \multirow[t]{3}{*}{$\begin{array}{l}\text { FFSF } \\
\text { (9 meses) }\end{array}$} & OSN bebê $1^{\circ}$ episódio (educ) & $\begin{array}{l}\text { rhô } \\
p\end{array}$ & $\begin{array}{l}0,769^{*} \\
0,018\end{array}$ & $\begin{array}{l}0,699 \\
0,054\end{array}$ \\
\hline & $A^{+}$ & rhô & $0,853^{*}$ & \\
\hline & & $p$ & 0,015 & \\
\hline \multirow{4}{*}{$\begin{array}{l}\text { CITMI-R } \\
\text { (12 meses) }\end{array}$} & $\mathrm{S}^{+}$ & rhô & $0,811^{*}$ & \\
\hline & & $p$ & 0,027 & \\
\hline & \multirow[t]{2}{*}{ Total de interativos (educ) } & rhô & $0,800^{*}$ & \\
\hline & & $p$ & 0,031 & \\
\hline
\end{tabular}

Fonte: Elaborada pela autora. *A correlação é significativa no nivel 0,05 (2 extremidades). Dados obtidos a partir da correlação de Spearman.educ=educadora

\section{Discussão}

Com relação à qualidade da vinculação estabelecida, os dados sugerem que aos nove meses os bebês interagiram positivamente tanto com a mãe como com a educadora, nos dois episódios de interação $\left(1^{\circ}\right.$ e $3^{\circ}{ }^{\circ}$. Os bebês apresentaram maior capacidade de se autorregular em interação com a educadora, visto que na presença da mãe apresentaram mais comportamentos de protesto. Seixas et al.(2017) destacaram a relação entre a representação das mães sobre seus filhos diretamente relacionada a forma com a qual interagem com os mesmos. Como as mães consideraram os bebês com temperamento mais dificil, possivelmente responderam mais prontamente às suas solicitações, exigindo menos comportamentos de autorregulação. A relação de afeto estabelecida entre a diade também pode ser uma variável importante para a classificação do temperamento. Levanta-se a hipótese de que as mães, por interagirem com somente um bebê, tendem a responder prontamente às suas necessidades, reforçando seus comportamentos de protesto. No ambiente dos centros de educação infantil os bebês aprendem que seus comportamentos de protesto não são prontamente atendidos, reforçando seus comportamentos de autorregulação nesses contextos, uma vez que a cuidadora tem muitos bebês para atender. Também, a presença de mais comportamentos de autorregulação por parte dos bebês pode aumentar a possibilidade de que sejam considerados de temperamento fácil pelas cuidadoras. Observa-se que os bebês aprendem a responder diferentemente às duas situações, determinando como reagem à interação com cada cuidador (mãe e educadora) que determina como são avaliados por elas, em termos de temperamento.

A comparação dos episódios um e três do FFSF possibilitou identificar se o bebê após um episódio não interativo consegue retomar padrões de orientação social positiva próximos ao primeiro episódio, ainda que aumente a frequência de protestos (OSN) ou comportamentos não interativos (AUR) (Fuertes, 2004). No presente estudo, não foram observadas diferenças significativas tanto para OSP ou OSN, sugerindo que os bebês estabeleceram uma boa vinculação tanto com suas mães como com as educadoras, ou seja, há uma boa vinculação primária e secundária. Entretanto, os bebês procuraram mais por suas mães após o episódio não interativo 
enquanto em contrapartida apresentaram mais comportamentos autorregulatórios com as educadoras. Ao não responderem desta maneira com suas mães, sugere-se que elas atendam mais prontamente suas necessidades e que eles discriminam essa condição. Ao considerar que são capazes de discriminar essa condição e exigirem mais de suas mães, sugere-se que esse vínculo seja mais forte, corroborando os apontamentos de Farran e Ramey (1977). Porém, observa-se que, ainda assim, a qualidade do vínculo com a educadora é boa, sem enfraquecer o vínculo com a mãe, contrariando os achados de Caruso (1990) e corroborando os achados de Baumgartner, Bellucci e Laghi (2017), que indicaram a possibilidade de se estabelecer uma relação de vinculação segura com cuidadores alternativos sem prejudicar a vinculação com as figuras de vinculação primária.

A partir da perspectiva de desenvolvimento adotada no presente estudo, compreende-se que os processos de interação e os padrões comportamentais emitidos pelo bebê, em interação com sua mãe ou com a educadora, decorreram de processos de aprendizagem ocorridos na relação entre os dois (Linhares \& Martins, 2015). Os bebês demonstraram mais comportamentos de autorregulação após o episódio não interativo (Chiodelli, 2016) com suas educadoras do que com suas mães, sugerindo que nesse contexto tenham que adaptar-se mais à situação de espera. As educadoras, por sua vez, apresentaram maior repertório para promover essa espera sem que o mesmo se engaje em comportamentos de protesto (cantar, disponibilizar brinquedo ou alimento, promover condições para que o bebê se distraia até ser atendido).

As mães, no contexto individualizado, podem desenvolver outros comportamentos responsivos que sinalizem para o bebê maior probabilidade de ser atendido mediante verbalização, choro e manifestação de desconforto, exigindo desse modo, menos comportamentos autorregulatórios (Seixas et al. 2017). Para Montiroso et al. (2010) altos niveis de comportamentos autorregulatórios associam-se a maior estresse percebido no episódio não interativo, levando a maior atraso para recuperar a qualidade da interação inicialmente obtida.

Considerando que, para essa amostra, mães e educadoras emitiram comportamentos de forma semelhante no FFSF, sugere-se que essa diferença se dê também pela forma diferente que mães e educadoras perceberam o temperamento dos próprios bebês (Fuertes et al., 2009; Linhares \& Martins, 2015; Seixas et al. 2017).

Jáaos doze meses, os bebês pareceram responder de modo semelhante tanto em interação com a mãe quanto com a educadora. As mães e as educadoras também continuaram emitindo comportamentos de modo semelhante. Aqui a hipótese poderia ser a presença dos brinquedos, que favoreceram a emissão de comportamentos não interativos em função da exploração do ambiente. Esse dado é importante para orientação aos pais sobre a importância da brincadeira no desenvolvimento infantil, com a manutenção de comportamentos de interação social positiva, porém, respeitando o espaço de exploração pelo bebê.

Quanto à percepção temperamental dos bebês, considerando que as mães indicaram o temperamento de seus filhos como mais dificeis do que as educadoras, possivelmente emitiram mais comportamentos de orientação positiva. Para Seixas et al. (2007) as relações de afeto positivo materno podem influenciar comportamentos autorregulatórios do bebê, com menor apresentação de comportamentos de protesto no episódio não interativo do FFSF. Também há que se considerar o fato de que as educadoras possuem modelos para comparar os bebês uns com os outros, e podem estar mais preparadas para lidar com as questões pertinentes ao desenvolvimento (Silva, 2018). A percepção do temperamento pela educadora, mostrou-se correlacionada aos comportamentos dos bebês aos nove meses e aos doze, sugerindo que as educadoras conseguiram responder aos bebês de modo individual, percebendo caracteristicas particulares dos bebês (ou responderam em função da sua percepção). Amostras maiores podem encontrar correlações também entre o temperamento indicado pela mãe 
conforme achados de outros estudos (Chiodelli, 2016; Fuertes et al., 2009).

\section{Considerações finais}

Os dados sugerem que os bebês da presente amostra apresentaram boas condições para estabelecer processos de vinculação com pessoas que se mostrem disponiveis para interação no ambiente e que lhes possa assegurar as condições de segurança, cuidado e afeto. Essas interações passam a ter sentido intencional, podendo formar-se relações mais complexas.

Dessa forma, os dados obtidos sugerem que, observadas as condições de constância da educadora e preparo para reconhecimento de seu potencial frente aos desafios da educação infantil (Silva, 2018) a transição para os cuidados oferecidos por outros pode ser realizada sem prejuízo de vínculo primário (vinculação com a mãe/pai) e com boas condições de vínculo secundário (educadora/outros adultos constantes e afetivos na vida do bebê). Porém, há limitações do estudo em relação ao número limitado da amostra, o que poderá ser confirmado e ampliado em futuros estudos com populações maiores.

Em relação à vinculação é possivel sugerir que na medida em que aumentam as capacidades de autorregulação da criança, o processo de socialização com outras pessoas também é favorecido. Sobretudo, é importante ressaltar que as relações de vinculação estabelecidas entre a criança e seu cuidador, seja no contexto primário ou secundário, influenciam o desenvolvimento emocional e cognitivo da criança, conforme já indicado por Saur et al. (2018). Portanto, a transição de cuidados na infância, para além da oferta de ambiente seguro e estruturado, deverá observar a qualidade das relações que se estabelecem no ambiente infantil, promovendo acolhimento, responsividade e condições para autorregulação.

\section{Referências}

Alvarenga, P., \& Cerezo, M. A. (2013). Interação mãe-criança : fidedignidade da versão brasileira do sistema observacional CITMI-R1. Avaliação Psicológica, 12(71), 307-316. DOI INEXISTENTE.
Ainsworth, M. D. S., Blehar, M. C., Waters, E., \& Wall, S. (1978). Patterns of attachment: A psychological study of the strange situation. Hillsdale: Erlbaum.

Bowlby, J. (1989). Uma base segura: aplicações clinicas da teoria do apego. Porto Alegre: Artes Médicas.

Cárcamo, R. A., García-Riquelme, A., Lagos, S., \& Gómez, E. (2019). Apego en niños con asistencia temprana a salas cuna en la ciudad de Punta Arenas (Chile): un estudio piloto. Rev. Lat Inoam.Cienc. Soc.Niñez, 17(2), 1-21. https://doi.or$\mathrm{g} / 10.11600 / 1692715 \times .17210$

Caruso, D. A. (1990). Infant Day Care and the Concept of Developmental Risk. Infant Mental Health Journal, 11(358-364). https://doi.org/10.1002/1097-0355(199024)11:4<358::AID-IMHJ2280110408>3.0.CO:2-C

Chiodelli, T. (2016). Temperamento e prematuridade: influências sobre a interação mãe-bebê. (Dissertação de mestrado). Universidade Estadual Paulista Julio de Mesquita Filho, Bauru, São Paulo, Brasil.

Faria, A., \& Fuertes, M. (2007). Reactividade infantil e a qualidade da interacção mãe-filho. Análise Psicológica, 4, 613-623. https://doi.org/10.14417/ap.470

Farran, D. C., \& Ramey, C. T. (1977). Infant Day Care and attachment behaviors toward mothers and teachers. Child Development, 48, 1112-1116. https://doi. org/10.2307/1128371

Favaro, M. de S. F., Peres, R. S., \& Santos, M. A. dos. (2012). Avaliação do impacto da prematuridade na saúde mental de puérperas. Psicologia USP, 17, 457-465. https://doi.org/10.1590/S1413-82712012000300012

Fuertes, M. (2004). Rotas da vinculação: O desenvolvimento do comportamento interactivo e a organizacao da vinculação no primeiro ano de vida do bebé prematuro. (Dissertação de Mestrado). Universidade do Porto, Porto, Portugal.

Fuertes, M., Faria, A., Soares, H., \& Oliveira-Costa, A. (2010) Momentos de interação em que as emoções se apre(e) ndem: estudo exploratório sobre a prestação materna e infantil em jogo livre. Psicologia USP, 21(4), 833-857. https://doi.org/10.1590/S0103-65642010000400010

Fuertes, M., Lopes-Dos-Santos, P., Beeghly, M., \& Tronick, E. (2009). Infant coping and maternal interactive behavior predict attachment in a Portuguese sample of healthy preterm infants. European Psychologist, 14(4), 320-331. https://doi.org/10.1027/1016-9040.14.4.320

Gonçalves, I., \& Fuertes, M. (2015). A Importância do Pai na Qualidade da Autorregulação do Bebé. In: Fuertes, M., Nunes, C, Rosa, J. (Orgs) Evidências Em Intervenção Precoce. Lisboa: Centro de Estudos Educacionais, 37-54.

Linhares, M. B. M., \& Martins, C. B. S. (2015). O processo da autorregulação no desenvolvimento de crianças. Estudos de Psicologia, 32(2), 281-294. https://doi. org/10.1590/0103-166X2015000200012

Maia, J. M. D., \& Williams, L. C. de A. (2005). Fatores de risco e fatores de proteção ao desenvolvimento infantil: uma revisão da área. Temas Em Psicologia, 13(2), 91-103. 
Malhado, S.C.B.; Alvarenga, P (2012). Relações entre o temperamento infantil aos oito meses e as práticas educativas maternas aos 18 meses de vida da criança, Estudos de Psicologia, Campinas, 29(Supl.), 789-797. https://doi.org/10.1590/S0103166X2012000500015

Massie, H., \& Campbell, B. K. (1983). The Massie-Campbell scale of mother-infant attachment indicators during stress. En J. Call, E. Galenson, \& R. Tyson (Orgs.), Frontiers of infant psychiatry. Nueva York: Basic Books, 394-412

Montirosso, R., Borgatti, R., Trojan, S., Zanini, R., \& Tronick, E. (2010). A comparison of dyadic interactions and coping with still-face in healthy pre-term and full-term infants. British Journal of Developmental Psychology, 28(2), 347-368. https://doi. org/10.1348/026151009X416429

Nardi, C. G. de A., Rodrigues, O. M. P., Melchiori, L. E., Salgado, M. H., \& Tavano, L. D. (2015). Bebês com Sequência de Pierre Robin : saúde mental materna e interação mãe-bebê. Estudos de Psicologia, 32(1), 129-140. https://doi.org/10.1590/ 0103-166X2015000100012

Pereira, V. A., Rodrigues, O. M. P., Carvalho, S. Z. L. de, \& Chiodelli, T. (2015). Influências do Estresse e Ansiedade Puerperal nos Primeiros Meses do Desenvolvimento Infantil. Cadernos de Pós-Graduação Em Distúrbios Do Desenvolvimento, 15(1), 89-100. DOI INEXISTENTE

Roecker, S., Marcon, S. S., Decesaro, M. das N., \& Waidman, M. A. P. (2012). Significados E Percepções Sobre Centro De Educação Infantil Mother - Child Relation Under the Light of the attachment theory: Revista de Enfermagem UERJ, 20(1), 27-32. DOI INEXISTENTE

Sanson, A., Hemphill, S. A., \& Smart, D. (2004). Connections between temperament and social development: a review. Social Development, 13(1), 142-166, https://doi.org/10.1046/j.1467-9507.2004.00261.X

Saur, B., Bruck, I., Antoniuk, S. A., \& Riechi, T. I. J. de S. (2018). Relação entre vínculo de apego e desenvolvimento cognitivo, linguístico e motor. Psico, 49(3), 257. https://doi.org/10.15448/1980-8623.2018.3.27248

Seixas, İ., Barbosa, M., \& Fuertes, M. (2017). Contributos para a auto-regulação do bebé no Paradigma Face-to-Face Still-Face. Análise Psicológica, 35(4). 469-485. https://doi.org/10.14417/ap.1280

Senicato, C., Lima, M. G., \& Barros, M. B. de A. (2016). Ser trabalhadora remunerada ou dona de casa associa-se à qualidade de vida relacionada à saúde? Cadernos de Saúde Pública, 32(8), 1-12. https://doi. org/10.1590/0102-311X00085415

Silva, V. dos R. (2018). O que pensam as educadoras e o que nos revelam os bebês sobre a organização dos espaços na educação infantil. (Dissertação de Mestrado). Universidade Federal de Sergipe. Brasil.

Thomas, A., \& Chess, S. (1977). Temperament and development. New York: Pleno.
Tronick, E., Als, H., Adamson, L., Wise, S., \& Brazelton, B. (1978). The infant's response to entrapment between contradictory messages in face-to-face interaction. American Academy of Child Psychiatry, 8(3), 1-13. https://doi.org/10.1016/So002-7138(og)62273-1

\section{Isabela Rocha Izidoro}

Mestre em Psicologia pela Universidade Federal da Grande Dourados (UFGD).

\section{Veronica Aparecida Pereira}

Doutora em Educação Especial, com pós-doutorado em Psicologia do Desenvolvimento. Docente do curso de Psicologia e do Programa de Pós-Graduação em da Universidade Federal da Grande Dourados (UFGD)

\section{Olga Maria Piazentin Rolim Rodrigues}

Doutora em Psicologia Experimental. Docente do curso de Psicologia e do Programa de Pós-Graduação de Psicologia do Desenvolvimento e Aprendizagem da Universidade Estadual Paulista Júlio de Mesquita Filho - Unesp-Bauru.

\section{Endereço para correspondência}

Veronica Aparecida Pereira - UFGD - Unidade II - Faculdade de Ciências Humanas - Programa de Pós-Graduação em Psicologia - Rodovia Dourados/Itahum, km 12 - Caixa Postal 364 - Dourados MS - CEP 79804-970 